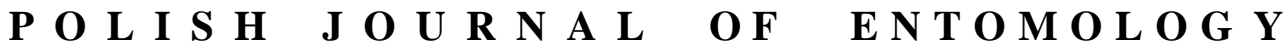

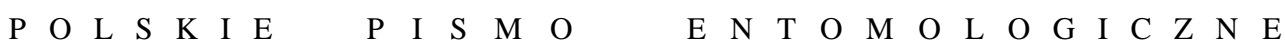

VOL. 84: 101-126

Lublin

30 June 2015

DOI: $10.1515 /$ pjen-2015-0009

\section{Relationships between bees (Hymenoptera: Apoidea: Apiformes) and flowers in the Bulgarian agricultural landscape}

\author{
JÓZEF BANASZAK ${ }^{1}$, PIOTR SZEFER $^{1 *}$, BOJANA DOCHKOVA ${ }^{2}$ \\ ${ }^{1}$ Department of Ecology, Institute of Environmental Biology, Kazimierz Wielki University, \\ 12 Ossolińskich Av., PL-85-093 Bydgoszcz, Poland; e-mail:lednica@ukw.edu.pl, \\ szefer85@gmail.com \\ ${ }^{2} 23$ San Stefano, ap. 4, BU-5800 Pleven, Bulgaria; e-mail: b_dochkova@abV.bg
}

\begin{abstract}
The species composition and number of visitations of food plants by bees were studied in refuge sites in agricultural landscapes and in selected crops. The habitat fragments of interest are characterised in terms of pollinator diversity at genus level and the use of food plants by individual genera. Trophic and temporal niche overlap is described for individual genera and the honey bee Apis mellifera in different habitat types. Factors influencing the manner of use of individual plant species by pollinating insects are identified.
\end{abstract}

KEY WORDS: Hymenoptera, Apoidea, Apiformes, Bulgaria, Pleven Region, Plovdiv Region, species diversity, melliferous plants, agricultural landscape.

\section{INTRODUCTION}

Studies have repeatedly confirmed the great importance of refuge habitats for wild bees (BANASZAK 1983, BOULINGER et al. 1998, MARSHALl 2004, HolzSCHUH et al. 2012, LentinI et al. 2012, MoRANDin \& KREMEN 2013, KENNEDY et al. 2013). The food resources of wild bees (and the honey bee Apis mellifera LiNNAEUS, 1758) constitute an important element in planning the conservation of these valuable insects. In this context, it is useful to understand and identify factors influencing the choice of plants by individual

* present address: Dr. Jana Biziela Str. 22/8, Bydgoszcz PL-85-163, Poland 
species and to determine how changes in resource availability influence pollinator populations.

Owing to its location, considerable terrain diversity and climate, Bulgaria offers particularly favourable conditions for bee keeping and the richness of the wild bee fauna. The natural base is formed by a wealth of food plants. Melliferous plants, whose flowers are natural food sources for the honey bee and wild bees in Bulgaria, are represented by 1020 species from 296 genera (TASHEV \& PANCHEVA 2009, 2011).

Older faunal papers from Bulgaria, dating mainly from the 1960s, contain data on the bees' food plants. This information, which we owe mainly to Prof. Neno ATANASSOV, is, however, mainly narrow in scope as regards identification of the bee fauna of Bulgaria and contributes to our knowledge about plant species visited by bees to a similar extent (ATANASSOV 1960, 1962a, 1960b, 1965, 1972a, 1972b, 1974). The 1980s, in turn, witnessed utilitarian research, focusing on pollinators of selected crops, mainly alfalfa (e.g. DochKova 1981a, 1981b, 1982a, 1982b, Dimitrov 1990; Dimitrov \& Dimitrova 1991a, 1991b).

At the same time, there has been a dearth of research on the patterns of use of wild plants by bees in an agricultural landscape in refuges of wild bees. i.e. at the edges of fields, roadsides and in small mid-field clumps of trees. Although there is research showing that wild flowers in semi-natural habitats are an irreplaceable food source for wild bees (KLEIN et al. 2012, MANDELIK 2012, LE FÉON et al. 2013, RoLLIN et al. 2013), little insight has been gained into the floral preferences of individual genera of wild bees within entire insect and plant communities (TUELL et al. 2008). The present study attempts to fill this void. The research questions for this study were as follows: 1) What are the patterns in plant visitations by bees, i.e. in their diets and diversity in the landscape? 2) Which pollinator groups are more frequently encountered in crops and in refuge habitats?

There are a large number of melliferous plants in Bulgaria, with 564 species from 232 genera and 75 families (TASHEV \& PANCHEVA 2009), compared to the total of 1,010 species recorded in this country (TASHEV \& PANCHEVA 2011). The present study also provides abundant data about the relations between bees and flowers. It adds to the body of data on the trophic base of the honey bee and wild bees in refuge habitats of Apiformes in the agricultural landscape of Bulgaria. It contains information about 104 species of wild plants. This is an important contribution, considering that the area of agricultural land in Bulgaria is $6,845.8$ ha or $61.6 \%$ of the total area (NPGR 1995). While previous studies of wild bees did contain species lists (e.g. ATANASSOV 1960, 1962a, 1962b, 1965, 1972a, 1972b, 1974 and other minor publications), they were mostly concerned with areas of natural habitats in Bulgaria. The present list contains data relating exclusively to refuge habitats (crop field margins), which accounts for the novelty of the data. 
This paper is an analysis of the composition and number of visitations of food plants by bees. The habitat fragments of interest are characterised in terms of pollinator diversity at genus level and the use of food plants by individual genera. Trophic and temporal niche overlap is described for individual genera and the honey bee in different habitat types. Factors influencing the manner of use of individual plant species by pollinating insects are identified.

\section{MATERIALS AND METHODS}

The field research was conducted at 15 sites. Some of them were marginal habitats: a field and ditch edges, covering small areas, usually 1-3 m wide belts dominated by ruderal plants with some species characteristic of xerothermic grasslands and meadows. Larger areas were covered by grasslands along the Vit river valley, with mostly thermophilous vegetation, bordering on fields. Fields of 5 selected crops were also investigated. Individual sites were sampled in the years 1987-1991, usually during two or three growing seasons (from spring to autumn). A total of 10 natural habitats were analysed (the site codes are given in brackets): valley of the River Vit (val_vit) - sampled from 1987 to 1989; roadside in the Pleven Region (road_ple) - sampled from 1987 to 1989; irrigation ditch near maize Zea mays L. (d_maize) - sampled from 1987 to 1989; ditch edge (dit_edg) - sampled in 1987 and 1990; slope of the Vit Valley (vit_slo) - sampled from 1988 to 1989; alfalfa field margin (alf_marg) - sampled from 1987 to 1989); slope of the hill (slope) - sampled only in 1988; railway embankment (rail_emb) - sampled from 1987 to 1989; roadside in Plovdiv (road_plo) - sampled in 1990-1991; ditch edge in Plovdiv (d_edge_plo) - sampled from 1990 to 1991. Fields of the following five crops were also sampled: Alfalfa Medicago sativa L. (alfalfa) from 1987 to 1991; Sunflower Helianthus annuus L. (sunflo) from 1988 to 1989; Oilseed rape Brassica napus L. (bra_nap), 'Elena', 'Tobin', and a Canadian cultivar from 1990 to 1991; Radish Raphanus sativus L. 'Omega' (radish) from 1990 to 1991; Phacelia Phacelia tanacetifolia BENTH. (phacelia) in 1991.

Bees were sampled, usually at two-week intervals, during the growing season in the years 1987-1991. A $200 \mathrm{~m}$ long and $1 \mathrm{~m}$ wide transect was marked in each of the 15 study sites (BANASZAK 1980) in order to record bee species visiting flowers and identify them live as to genus (wild bees) or species (honey bees and some bumblebees). The researcher walked slowly along the transect counting and gathering every specimen of solitary bees and counting bumblebees and honey bees. If a specimen was caught on a flower species, it was considered to be a food relationship (nectar or pollen). Bee counts from each transect were then calculated into densities expressed as individuals $* \mathrm{ha}^{-1}$. We used raw bee-plant relations in the analysis of niche breadth. Prior to numerical analysis (NMDS, MRPP), the 
visitations were calculated into the expected number of visitations if the flower species was present in the transect. The gathered specimens were identified to species level: the results are presented in another paper (BANASZAK \& DocHKOVA 2014). The food plant species visited were also noted for each bee record so that a list of food plants of bees from the study habitats could be compiled.

Nonmetric multi-dimensional scaling (NMDS), a non-parametric ordination method, was used to order the sites with regard to pollinator activity, i.e. estimated expected annual visitation counts for every bee genus present at that site. The Bray-Curtis distance was used as the measure of distance. The non-parametric permutation test MRPP was carried out in order to verify the significance of element grouping in the ordination plot (ZIMMERMAN et al. 1985). The IndVal method (DUFRENE \& LEGENDRE 1997) was employed to identify the activities underlying the significance of these clusters. The NMDS coordinates for the study sites along either axis were subsequently associated with their characteristics so as to identify correlations between them and changes in pollinator activity consistent with the NMDS plot. As the distribution of the variables was not normal, Spearman's nonparametric rank correlation was used to test for the statistical significance of relations between habitat characteristics and the configuration obtained by NMDS.

Trophic niche breadth (AGUIAR et al. 2012) was determined for each genus using Shannon's Index of diversity (SHANNON 1948) for percentages of individual food plants, according to the formula below:

$$
H^{\prime}=\sum_{i=1}^{S} \ln \left(p_{i}\right) \times p_{i},
$$

where $p_{i}$ is the proportion of the $i^{\text {th }}$ species in an assemblage.

The degree of trophic and temporal niche overlap was determined by calculating Czekanowski's Index (FEINSINGER et al. 1981) for all pairs of genera, according to the formula:

$$
O_{12}=O_{21}=1-0.5 \sum_{i=1}^{n}\left|p_{i 1}-p_{i 2}\right|,
$$

where $p_{i 1}$ and $p_{i 2}$ are the proportions of the $1^{\text {st }}$ and $2^{\text {nd }}$ bee species in the use of the $i^{\text {th }}$ floral resource. It is an easy measure of the area of overlap between two distributions of use of particular resource classes. It assumes a value of 0 when the distributions of resource use are completely separate and a value of 1 when they are identical.

The distributions of bee visitations of individual plant species were compared with the Kolmogorov-Smirnov test for two independent samples (SIEGEL 1956).

A trophic niche overlap analysis based on the null model (GOTELLI \& GRAVES 1996) was carried out in order to determine whether competition had a significant impact on the pattern of visitations in the bee assemblage. The null model tests whether an observed 
pattern of niche overlap is significantly different from one generated by a random resource use assembly for a given assemblage. Niches were analysed with regard to their food resources and period of occurrence. For the former, the EcoSim software package (GOTELLI \& ENTSMINGER 2001) and the RA2 algorithm (LAWLOR 1980) were used. The algorithm substitutes random numbers from 0 to 1 for actual resource use values, thus partly preserving niche breadth for a particular species. Time overlap of niches was studied with TimeOverlap software and the Rosario algorithm (CASTRO-ARELLANO et al. 2010), which, unlike other algorithms, preserves most autocorrelations in time series. In both cases, the number of randomisations was 1000 for each site and simulation.

\section{RESULTS}

\section{List of food plants}

A total of 37038 records of flower visitations by bees were collected over the five years of study, of which $83.2 \%$ (30805) were by the honey bee. The 15 study sites yielded a total of 233 species representing 23 genera of wild bees. The inventory can be found in an earlier paper (BANASZAK \& DOCHKOVA 2014). Most records concerned the genera Halictus LATREILLE, 1804 (sensu lato) - 3057 (8.2 \%), Andrena FABRICIUS, 1775 - 1159 (3.1\%) and Eucera SCOPOLI, 1770 - $496(1.3 \%)$. The remaining records were of the genera Bombus LATREILle, 1802 (205), Megachile LATREILle, 1802 (192), Systropha ILliger, 1806 (191), Rophitoides SCHENCK, 1861 (153), Rophites SPINOLA, 1808 (127), Nomada SCOPOl, 1770 (109), Osmia PANZER, 1806 (92), Dasypoda LATREILle, 1802 (71), Anthidium FABricIUS, 1804 (59), Hylaeus FABricIUS, 1793 (41), Ceratina LATREILLE, 1802 (12). The genera Anthophora LATREILle, 1803, Heriades SPINOLA, 1808, Coelioxys Latreille, 1809, Colletes Latreille, 1802, Meliturga Latreille, 1827, Sphecodes LATREILLE, 1806 and Xylocopa LATREILLE, 1802 were each recorded 10 times or more rarely and were therefore excluded from subsequent numerical analyses in order to avoid a low abundance effect.

A total of 100 species of plants visited by bees were identified collectively in all the study transects (Tab. 1). Among the semi-natural habitats, the highest number of food plant species visited by bees was noted for the Ditch near maize (43) site and the lowest for the Slope of the Vit Valley (18) (Tab. 2). Plants visited by the largest number of species included Carduus acanthoides L. (15 bee genera), Medicago sativa L. (13 genera), Cichorium intybus L., Echium vulgare L., Lamium purpureum L. and Malva silvestris L. (10 genera). 
Tab. 1. List of food plant species for wild bees found in research plots. Each value in the the expected number of visits in one year on a given plant species. Sites: 1 - rape field, 6 - ditch edge, 7 - Phacelia field, 8 - roadside Pleven, 9 - alfalfa field margin, 10 - radish 14 - slope of the hill, 15 - road-side Plovdiv.

\begin{tabular}{|c|c|c|c|c|c|}
\hline \multirow{3}{*}{$\begin{array}{l}\text { Plant species } \\
\end{array}$} & \multirow[b]{2}{*}{1} & \multirow[b]{2}{*}{2} & \multirow[b]{2}{*}{3} & \multirow[b]{2}{*}{4} & \multirow[b]{2}{*}{5} \\
\hline & & & & & \\
\hline & 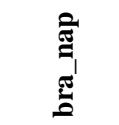 & $\begin{array}{l}\vec{\nabla} \\
\frac{\vec{J}}{D}\end{array}$ & 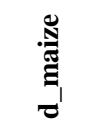 & $\frac{\frac{\pi}{\sigma}}{\frac{\pi}{\sigma}}$ & 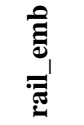 \\
\hline \multicolumn{6}{|l|}{ Achillea millefolium $\mathrm{L}$. } \\
\hline \multicolumn{6}{|l|}{ Ajuga genevensis L. } \\
\hline Alcea rosea $\mathrm{L}$. & & & 13.00 & & \\
\hline \multicolumn{6}{|l|}{ Alkanna strybnyi VEL. } \\
\hline Anchusa italica RETZ. & & 0.33 & & & \\
\hline Anchusa officinalis L. & & & & & 2.00 \\
\hline \multicolumn{6}{|l|}{ Anthemis arvensis $\mathrm{L}$. } \\
\hline \multicolumn{6}{|l|}{ Antirrhinum orontium $\mathrm{L}$. } \\
\hline Brassica napus L. var. oleifera & 6890.00 & & & & \\
\hline Calepina corvini DESV. & & 1.33 & & & \\
\hline \multicolumn{6}{|l|}{ Calepina irregularis THELL } \\
\hline \multicolumn{6}{|l|}{ Camelina sativa (L.) CRANTZ } \\
\hline Capsella bursa-pastoris (L.) MED. & & 3.00 & 2.67 & & \\
\hline Carduus acanthoides $\mathrm{L}$. & & 249.67 & 146.33 & & 24.00 \\
\hline Caucalis daucoides $\mathrm{L}$. & & 1.00 & 21.67 & & 1.50 \\
\hline \multicolumn{6}{|l|}{ Centaurea acanthoides $\mathrm{L}$. } \\
\hline \multicolumn{6}{|l|}{ Centaurea calcitrapa $\mathrm{L}}$. \\
\hline Centaurea orientalis $\mathrm{L}$. & & 0.67 & 1.33 & & \\
\hline \multicolumn{6}{|l|}{ Centaurea salonitana VIS } \\
\hline \multicolumn{6}{|l|}{ Chrysanthemum leucanthemum $\mathrm{L}$. } \\
\hline Cichorium intybus $\mathrm{L}$. & & 11.00 & 7.00 & & 3.50 \\
\hline \multicolumn{6}{|l|}{ Cirsium arvense L. (SCOP.) } \\
\hline Conium maculatum $\mathrm{L}$. & & & 1.00 & & \\
\hline Convolvulus arvensis $\mathrm{L}$. & & 1.33 & 6.33 & & 44.00 \\
\hline \multicolumn{6}{|l|}{ Coronilla varia $\mathrm{L}$. } \\
\hline Crepis paludosa (L.) MOENCH & & & & & 1.00 \\
\hline Crepis pulchra $\mathrm{L}$. & & & 3.33 & & 4.50 \\
\hline \multicolumn{6}{|l|}{ Crepis vireas $\mathrm{L}$. } \\
\hline Delphinium consolida $\mathrm{L}$. & & & 0.33 & & \\
\hline \multicolumn{6}{|l|}{ Delphinium nemorosa GAY } \\
\hline \multicolumn{6}{|l|}{ Delphinium orientale J. GAY } \\
\hline \multicolumn{6}{|c|}{ Descurainia sophia (L.) WEBB ex PRANTL } \\
\hline Echium vulgare $\mathrm{L}$. & & 128.00 & & & 1.00 \\
\hline Erodium cicutarium (L.) L'HER. & & 7.33 & 0.67 & & 2.50 \\
\hline Eryngium campestre L. & & & 2.33 & & \\
\hline Euphorbia cyparissias L. & & & 0.33 & & 5.50 \\
\hline
\end{tabular}


table is the mean number of observations for the sampled years and can be interpreted as 2 -valley of the Vit river, 3 - ditch near maize, 4 - alfalfa field, 5 - railway embankment, field, 11 - ditch edge Plovdiv, 12 - sunflower field, 13 - slope of Vit river valley,

\begin{tabular}{|c|c|c|c|c|c|c|c|c|c|}
\hline \multicolumn{10}{|c|}{ Site } \\
\hline 6 & 7 & 8 & 9 & 10 & 11 & 12 & 13 & 14 & 15 \\
\hline 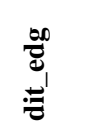 & 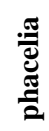 & 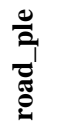 & 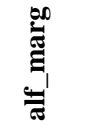 & 㺼 & 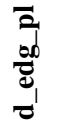 & $\stackrel{0}{\Xi}$ & $\begin{array}{l}\frac{0}{\sigma_{2}} \\
\frac{\overrightarrow{1}}{2}\end{array}$ & $\frac{\check{O}}{\frac{0}{n}}$ & 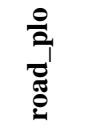 \\
\hline 3.00 & & 0.33 & 4.67 & & 1.00 & & 4.50 & & \\
\hline 2.00 & & & & & & & & & \\
\hline \multirow[t]{5}{*}{0.50} & & & 0.33 & & & & 2.50 & 8.00 & \\
\hline & & & & & 1.00 & & & & \\
\hline & & & & & & & 3.00 & 16.00 & \\
\hline & & & & & & & & & \\
\hline & & & & & 0.50 & & & & \\
\hline \multicolumn{10}{|l|}{0.50} \\
\hline & & 61.67 & & & & & & & \\
\hline & & 0.67 & & & 3.50 & & & & 4.00 \\
\hline & & 3.00 & & & 2.00 & & & 3.00 & 10.00 \\
\hline & & & & & 2.00 & & & & \\
\hline & & 2.00 & 1.67 & & 0.50 & & & & \\
\hline 463.00 & & 49.00 & 560.67 & & 15.50 & & 393.50 & 257.00 & 115.50 \\
\hline \multirow[t]{3}{*}{0.50} & & & 0.33 & & 0.50 & & & 1.00 & \\
\hline & & & & & & & & & 2.00 \\
\hline & & & & & & & 1.50 & & \\
\hline \multirow[t]{3}{*}{0.50} & & 2.00 & 12.33 & & & & 14.00 & & 3.00 \\
\hline & & 30.33 & 8.33 & & 65.00 & & & & 78.50 \\
\hline & & 0.67 & & & & & & & \\
\hline \multirow[t]{2}{*}{7.50} & & 22.67 & 8.67 & & 1.00 & & 4.50 & 19.00 & 2.00 \\
\hline & & & & & & & & & 0.50 \\
\hline & & & & & & & & & \\
\hline 66.50 & & 20,00 & 5.00 & & 11.50 & & & 14.00 & 14.00 \\
\hline \multirow[t]{2}{*}{6.50} & & & 0.67 & & & & 0.50 & & \\
\hline & & & & & 1.00 & & & & 0.50 \\
\hline \multirow[t]{6}{*}{3.50} & & 0.67 & 0.33 & & 9.50 & & 1.00 & 10.00 & \\
\hline & & & & & 3.00 & & & & \\
\hline & & 0.33 & 19.00 & & 1.00 & & 3.00 & & \\
\hline & & 0.33 & & & & & & & \\
\hline & & 4.33 & 19.00 & & & & 0.50 & & \\
\hline & & & & & 1.00 & & & & 1.50 \\
\hline \multirow[t]{4}{*}{10.50} & & 55.33 & & & & & 1.50 & & \\
\hline & & 5.33 & 1.00 & & & & & & 1.00 \\
\hline & & & & & & & & & \\
\hline & & 0.67 & 0.67 & & 1.00 & & & 1.00 & \\
\hline
\end{tabular}


Tab. 1. Continued.

\begin{tabular}{|c|c|c|c|c|c|}
\hline \multirow{3}{*}{$\begin{array}{ll}\text { Plant species } \\
\end{array}$} & \multirow[b]{2}{*}{$\mathbf{1}$} & \multirow[b]{2}{*}{2} & \multirow[b]{2}{*}{3} & \multirow[b]{2}{*}{4} & \multirow[b]{2}{*}{5} \\
\hline & & & & & \\
\hline & 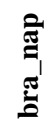 & $\begin{array}{l}\vec{\nabla} \\
\frac{\vec{z}}{\partial}\end{array}$ & 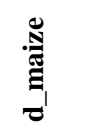 & 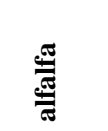 & 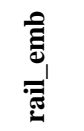 \\
\hline \multicolumn{6}{|l|}{ Euphorbia seguierana NECKER } \\
\hline \multicolumn{6}{|l|}{ Galium aparine $\mathrm{L}}$. \\
\hline \multicolumn{6}{|l|}{ Galium boreale L. } \\
\hline \multicolumn{6}{|l|}{ Galium mollugo L. } \\
\hline \multicolumn{6}{|l|}{ Glechoma hederacea $\mathrm{L}$. } \\
\hline \multicolumn{6}{|l|}{ Helianthus annuus L. } \\
\hline Heliotropium europaeum L. & & & 0.67 & & 12.00 \\
\hline \multicolumn{6}{|l|}{ Hypeocum grandiflorum BENTH. } \\
\hline \multicolumn{6}{|l|}{ Hypericum perforatum $\mathrm{L}$. } \\
\hline \multicolumn{6}{|l|}{ Hyssopus officinalis $\mathrm{L}$. } \\
\hline Isatis praecox $\mathrm{KIT}$ & & 0.33 & & & \\
\hline Knautia arvensis (L.) COULT. & & 1.33 & 2.33 & & 1.00 \\
\hline Lamium album $\mathrm{L}$. & & 3.67 & 1.00 & & \\
\hline Lamium purpureum $\mathrm{L}$. & & 12.00 & 3.00 & & 74.50 \\
\hline Lathyrus annuus L. & & & & & 1.00 \\
\hline Lathyrus aphaca L. & & & 3.00 & & \\
\hline Lathyrus erectus LAG. & & & 0.33 & & \\
\hline Lathyrus tuberosus L. & & & 0.33 & & \\
\hline Lepidium draba $\mathrm{L}$. & & 16.00 & 33.67 & & 10.00 \\
\hline \multicolumn{6}{|l|}{ Linaria vulgaris MILLER } \\
\hline Lotus corniculatus $\mathrm{L}$. & & 1.00 & & & \\
\hline Malva neglecta WALLR. & & 12.33 & & & \\
\hline Malva sylvestris L. & & 6.67 & & & \\
\hline Matricaria chamomilla L. & & 1.33 & & & \\
\hline \multicolumn{6}{|l|}{ Medicago falcata $\mathrm{L}$. } \\
\hline \multicolumn{6}{|l|}{ Medicago lupulina L. } \\
\hline Medicago sativa $\mathrm{L}$. & & & & 389.00 & \\
\hline Melilotus officinalis ( L.) PALLA & & & 8.67 & & \\
\hline Mentha arvensis L. ssp. arvensis & & 0.67 & & & \\
\hline \multicolumn{6}{|l|}{ Myosotis arvensis (L.) HILL. } \\
\hline Myosotis intermedia LINK. & & 1.00 & 0.33 & & \\
\hline Nigella arvensis $\mathrm{L}$. & & & 14.67 & & \\
\hline \multicolumn{6}{|l|}{ Onobrychis calcarea VANDAS } \\
\hline Onopordon tauricum WILLD & & 8.67 & & & \\
\hline Orlaya grandiflora & & & 2.00 & & \\
\hline \multicolumn{6}{|l|}{ Ornithogalum narbonense L. } \\
\hline Papaver rhoeas L. & & 1.33 & 16.00 & & 1.00 \\
\hline \multicolumn{6}{|l|}{ Phacelia tanacetifolia BENTHAM } \\
\hline Physospermum aquilegifolium КОСН & & & 0.67 & & \\
\hline
\end{tabular}




\begin{tabular}{|c|c|c|c|c|c|c|c|c|c|}
\hline \multicolumn{10}{|c|}{ Site } \\
\hline 6 & 7 & 8 & 9 & 10 & 11 & 12 & 13 & 14 & 15 \\
\hline \multirow[t]{2}{*}{$\begin{array}{l}\underbrace{00}_{0} \\
\vec{E}_{1}^{\prime} \\
:=\end{array}$} & $\begin{array}{l}\frac{\pi}{\pi} \\
\frac{\pi}{\pi} \\
\frac{\pi}{2}\end{array}$ & 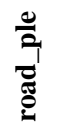 & 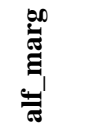 & $\frac{\bar{E}}{\overparen{Z}}$ & 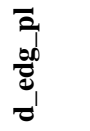 & $\stackrel{0}{\stackrel{\Xi}{\Xi}}$ & $\begin{array}{l}\frac{\theta}{\omega} \\
\dot{B} \\
\dot{\nabla}\end{array}$ & $\frac{\check{0}}{\omega}$ & 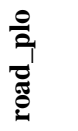 \\
\hline & & & & & 2.00 & & & & 0.50 \\
\hline 0.50 & & 0.33 & & & & & 0.50 & & \\
\hline \multicolumn{10}{|l|}{3.50} \\
\hline & & & & & & & 0.50 & & \\
\hline & & 0.33 & & & & & & & \\
\hline & & & & & & 1730.50 & & & \\
\hline \multirow[t]{2}{*}{3.50} & & & 0.67 & & & & & & \\
\hline & & & & & 0.50 & & & & \\
\hline \multirow[t]{3}{*}{1.00} & & & & & & & 1.50 & & \\
\hline & & & & & & & & 26.00 & \\
\hline & & 3.00 & & & & & & & \\
\hline 79.50 & & & 2.33 & & & & 1.00 & 43.00 & 1.00 \\
\hline 1.00 & & 14.67 & & & & & & & \\
\hline \multirow[t]{4}{*}{69.50} & & 73.00 & & & 47.00 & & & & \\
\hline & & & & & & & & & \\
\hline & & & & & & & & & \\
\hline & & 0.33 & & & & & & & \\
\hline & & & & & & & & & \\
\hline 185.50 & & 50.00 & 123.67 & & 1.50 & & & & \\
\hline \multicolumn{10}{|l|}{0.50} \\
\hline & & & & & & & & & 0.50 \\
\hline & & 3.67 & & & & & & & 2.00 \\
\hline & & 3.00 & & & 8.50 & & & & 24.00 \\
\hline & & & 0.67 & & 18.00 & & & & 1.00 \\
\hline & & 0.33 & & & & & & & \\
\hline & & & & & & & & & 32.50 \\
\hline 0.50 & & 0.67 & 0.33 & & & & & & \\
\hline \multicolumn{10}{|l|}{211.50} \\
\hline & & & & & & & 4.50 & 6.00 & \\
\hline \multicolumn{10}{|l|}{1.00} \\
\hline 0.50 & & 0.33 & 0.33 & & & & & & \\
\hline \multirow[t]{3}{*}{172.50} & & 0.67 & 2.33 & & 0.50 & & & & \\
\hline & & & & & & & & & 0.50 \\
\hline & & 10.67 & & & 15.00 & & & 1.00 & 41.00 \\
\hline \multirow[t]{5}{*}{4.50} & & 1.00 & & & & & & 1.00 & \\
\hline & & & & & & & 0.50 & & \\
\hline & & 31.33 & 3.33 & & & & 7.50 & & 5.50 \\
\hline & 931.00 & & & & & & & & \\
\hline & & & 0.33 & & & & 6.00 & & \\
\hline
\end{tabular}


Tab. 1. Continued.

\begin{tabular}{|c|c|c|c|c|c|}
\hline \multirow[t]{3}{*}{$\begin{array}{ll}\text { Plant species } \\
\end{array}$} & \multirow[b]{2}{*}{1} & \multirow[b]{2}{*}{2} & \multirow[b]{2}{*}{3} & \multirow[b]{2}{*}{4} & \multirow[b]{2}{*}{5} \\
\hline & & & & & \\
\hline & 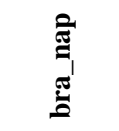 & $\begin{array}{l}\vec{z} \\
\frac{\vec{z}}{\pi}\end{array}$ & 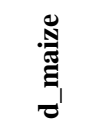 & 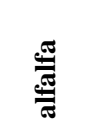 & 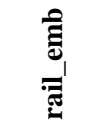 \\
\hline Plantago media L. & & 0.33 & & & \\
\hline Polygonum convolvulus L. & & & 0.67 & & 0.50 \\
\hline Polygonum persicaria L. & & 1.33 & & & \\
\hline Potentilla argentea $\mathrm{L}$. & & 2.00 & & & \\
\hline \multicolumn{6}{|l|}{ Prunus spinosa $\mathrm{L}$. } \\
\hline Ranunculus acris L. & & & 0.67 & & 0.50 \\
\hline Raphanus raphanistrum $\mathrm{L}$. & & 0.33 & 40.00 & & \\
\hline \multicolumn{6}{|l|}{ Raphanus sativus L. } \\
\hline Robinia hispida $\mathrm{L}$. & & & & & 2.00 \\
\hline Rosa canina $\mathrm{L}$. & & & 0.33 & & \\
\hline Rubus caesius $\mathrm{L}$. & & & 5.00 & & 10.00 \\
\hline \multicolumn{6}{|l|}{ Salvia aethiopis L. } \\
\hline Salvia nemorosa L. & & 2.33 & 0.67 & & \\
\hline \multicolumn{6}{|l|}{ Salvia pratensis $\mathrm{L}}$. \\
\hline \multicolumn{6}{|l|}{ Salvia verticillata $\mathrm{L}$. } \\
\hline Sambucus ebulus L. & & 5.67 & & & \\
\hline Senecio erucifolius $\mathrm{L}$. & & & 0.33 & & \\
\hline Silybum marianum (L.) GAERTNER & & 4.33 & & & \\
\hline Sinapis arvensis L. & & 4.67 & 11.33 & & \\
\hline \multicolumn{6}{|l|}{ Taraxacum hederifolia $\mathrm{L}$. } \\
\hline Taraxacum officinale WEBER (WIGGERS) coll. & & 2.33 & 0.67 & & \\
\hline \multicolumn{6}{|l|}{ Thymus marschallianus WILLD. } \\
\hline Tragopogon crucifolium $\mathrm{L}$. & & 0.33 & & & \\
\hline Tribulus terrestris $\mathrm{L}$. & & & & & 1.00 \\
\hline Trifolium repens $\mathrm{L}$. & & 2.33 & & & \\
\hline \multicolumn{6}{|l|}{ Triticum vulgare VILL. } \\
\hline Unidentified & & 7.00 & 6.67 & & 13.00 \\
\hline Verbascum nigrum L. & & 0.33 & & & 1.00 \\
\hline Verbascum sp. & & 0.33 & & & \\
\hline Verbena officinalis L. & & 2.00 & & & \\
\hline Veronica hederifolia $\mathrm{L}$. & & 0.33 & 0.67 & & \\
\hline Vicia cracca L. & & & 1.33 & & \\
\hline Vicia narbonensis L. & & & 0.33 & & \\
\hline Vicia striata $\mathrm{MB}$. & & & 0.33 & & \\
\hline Vicia villosa ROTH & & & 2.33 & & \\
\hline Viola arvensis MURRAY & & & 0.33 & & \\
\hline SUM & 6890.00 & 505.96 & 364.65 & 389.00 & 217.00 \\
\hline
\end{tabular}




\begin{tabular}{|c|c|c|c|c|c|c|c|c|c|}
\hline \multicolumn{10}{|c|}{ Site } \\
\hline 6 & 7 & 8 & 9 & 10 & 11 & 12 & 13 & 14 & 15 \\
\hline \multirow[t]{6}{*}{ 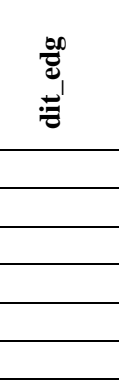 } & 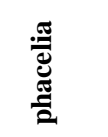 & 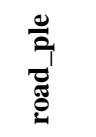 & 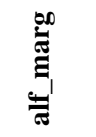 & 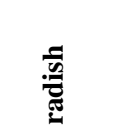 & $\begin{array}{l}\overline{\sigma_{1}} \\
\sigma_{0}^{0} \\
\sigma_{j}^{\prime} \\
\sigma^{\prime}\end{array}$ & $\stackrel{\varrho}{\stackrel{\Xi}{\Xi}}$ & $\begin{array}{l}\frac{0}{n_{1}} \\
\cdot \vec{b}\end{array}$ & $\frac{\check{0}}{\omega}$ & 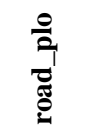 \\
\hline & & & & & & & & & \\
\hline & & & & & & & 200 & & \\
\hline & & 0.33 & & & & & & & \\
\hline & & 4.67 & & & & & & & \\
\hline & & & & & 1.50 & & & & \\
\hline \multirow[t]{3}{*}{3.00} & & 0.67 & 7.33 & & & & & & 1.00 \\
\hline & & & & 2172.00 & & & & & \\
\hline & & & & & 14.00 & & & & \\
\hline \multicolumn{10}{|l|}{1.50} \\
\hline \multirow[t]{2}{*}{3.00} & & 2.33 & & & 16.00 & & 3.00 & 1.00 & \\
\hline & & 0.67 & & & & & & & \\
\hline \multirow[t]{4}{*}{26.50} & & 51.33 & 9.33 & & & & 43.50 & 2.00 & \\
\hline & & & 3.33 & & & & & & \\
\hline & & 2.67 & & & & & & & \\
\hline & & & & & & & & 1.00 & \\
\hline 1.00 & & 0.33 & & & 2.00 & & 2.50 & & \\
\hline & & & & & & & & & \\
\hline \multirow[t]{6}{*}{36.00} & & 15.00 & 5.33 & & & & 1.00 & & \\
\hline & & & 0.33 & & & & & & \\
\hline & & & 0.33 & & & & & & 0.50 \\
\hline & & & & & & & 36.00 & & \\
\hline & & & & & & & & & 0.50 \\
\hline & & & & & & & & & \\
\hline \multicolumn{10}{|l|}{4.00} \\
\hline & & & & & 5.50 & & & & \\
\hline & & 3.67 & 0.33 & & 2.50 & & 3.50 & 29.00 & 10.00 \\
\hline & & & & & 0.50 & & & & \\
\hline & & & & & & & & & \\
\hline \multirow[t]{2}{*}{1.00} & & 0.33 & & & & & & & \\
\hline & & 6.67 & 0.67 & & & & & & \\
\hline \multicolumn{10}{|l|}{0.50} \\
\hline & & & & & & & & & \\
\hline & & & & & & & & & \\
\hline \multicolumn{10}{|l|}{0.50} \\
\hline 1376.50 & 931.00 & 521.33 & 803.64 & 2172.00 & 255.50 & 1730.50 & 543.50 & 439.00 & 353.00 \\
\hline
\end{tabular}




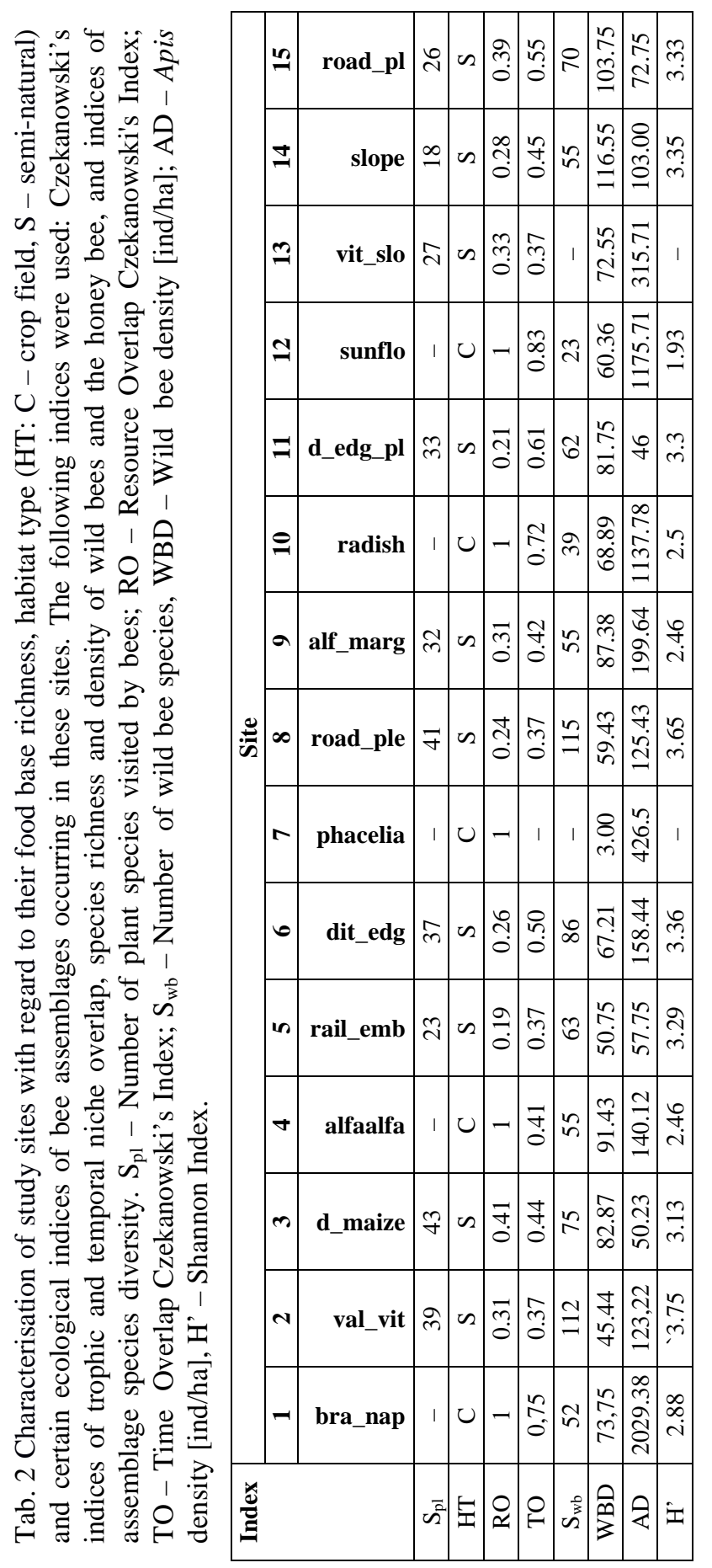




\section{Trophic niche breadth and niche overlap}

Diversification of the trophic base of individual bee species, reflected in the number of food plants, varied widely. The highest number of food plants was identified for Apis mellifera (75), while the genera Rophites and Rophitoides used only two food plants (Tab. 3). Plants of 11 species were visited only by the honey bee. The count of visitations of most of these species by other pollinators was low, an exception being Thymus marschalianus, which was intensively used. Apis mellifera was the dominant visitor to 22 food plant species visited also by other bees. For 15 plant species, half of the visitations recorded were by the honey bee, and 8 species accounted for $75 \%$ of visitations. Five food plant species were visited exclusively by the genus Halictus s.l., which accounted for $>50 \%$ of records on 7 plant species. The genus Eucera was also recorded exclusively on 5 plant species and the genera Nomada, Andrena and Megachile each visited only one plant species.

The honey bee topped the list in terms of the total visitations of flowers. $29.4 \%$ of food plant species intensively ( $>50 \%$ of the visitation count) foraged by Apis mellifera were also visited by representatives of other genera. Unlike the honey bee, the genera Rophites and Rophitoides used alfalfa nearly exclusively as their food source. The genus Osmia followed the most diversified diet, feeding rather evenly on 29 plant species. The most attractive food plant for bees was Carduus acanthoides, visited by 15 bee genera.

The genus Osmia had the most diversified diet $\left(\mathrm{H}^{\prime}=3.13\right)$, while the genera Rophites and Rophitoides had the narrowest niches $\left(\mathrm{H}^{\prime}=0.06\right.$ and $\left.\mathrm{H}^{\prime}=0,08\right)$. The diet of the honey bee was also not very much diversified $\left(H^{\prime}=1.42\right)$ as this species preferred to feed almost exclusively on flowers of oilseed rape, radish, sunflower and Phacelia spp. crops. Together, these plants accounted for nearly $95 \%$ of all plant visitations by the honey bee.

Czekanowski's Indices, reflecting the overlap of bees' trophic niches, ranged from almost 0 (completely separated niches) to almost 1 (identical niches) (Tab. 2). However, $75 \%$ of the values were low $(<0.25)$ and 16 were relatively high $(>0.5)$. The KolmogorovSmirnov test confirmed a fit between the distributions of food plant use for the following five pairs of taxa: Halictus s.l. and Andrena, Megachile and Anthidium, Osmia and Anthidium, Rhophites and Melitta KIRBY, 1802, and Rophites and Rophitoides. Dasypoda, Hylaeus and Bombus had the most unique food bases, demonstrating no high or significant overlap with any other genus analysed.

The genus Bombus, apart from the main food plants, such as Heliantus annuus or Phacelia tanacetifolia, would also more frequently visit plant species of the genus Delphinium L. These plants are less attractive to the other bee genera and the honey bee, with only Delphinium consolida being more frequently visited by Eucera and Hylaeus. Of great importance to Hylaeus were also plants rarely visited by other bee genera, mainly Mentha arvensis and Calepina corvin and, less so, Ranunculus acris. The genus Dasypoda 


\begin{tabular}{|c|c|c|c|c|c|c|c|c|c|c|c|c|c|c|c|c|c|}
\hline$I \stackrel{\infty}{=} 0$ & $\stackrel{n}{2}$ & 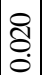 & 导 & ¿े & 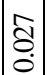 & $\underset{\substack{0 \\
\hdashline}}{\mid}$ & $\begin{array}{l}8 \\
0 \\
0 \\
0\end{array}$ & $\mid \begin{array}{c}8 \\
8 \\
0\end{array}$ & 8 & $\mid \begin{array}{l}0 \\
0 \\
0 \\
0\end{array}$ & & $\begin{array}{l}8 \\
8 \\
0 \\
0\end{array}$ & & 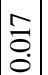 & & 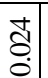 & \\
\hline $\overrightarrow{0} \overline{0} \tilde{0}$ & 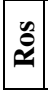 & $\begin{array}{c}\text { fo } \\
0 \\
0\end{array}$ & $\begin{array}{l}8 \\
8 \\
0\end{array}$ & $\begin{array}{l}0 \\
0 \\
0 \\
0\end{array}$ & 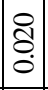 & 8 & $\begin{array}{l}8 \\
8 \\
0\end{array}$ & 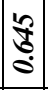 & $\frac{5}{0}$ & 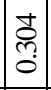 & ชิ & $\stackrel{\Xi}{\Xi}$ & 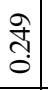 & \begin{tabular}{|}
8 \\
8 \\
0
\end{tabular} & 2 & 1 & - \\
\hline$\vec{F}$ & $\left|\begin{array}{c|}\tilde{2} \\
\simeq \\
\simeq\end{array}\right|$ & 导 & : & $\frac{\tilde{a}}{0}$ & $\overrightarrow{\mathrm{s}}$ & 官 & ठ̊. & $\mid$\begin{tabular}{l}
$\sqrt{6}$ \\
\hdashline \\
0
\end{tabular} & $\div$ & $\mid \begin{array}{c}8 \\
\tilde{m} \\
0\end{array}$ & हे & $\stackrel{5}{\Xi}$ & \begin{tabular}{|c|} 
\\
\multirow{2}{*}{} \\
0
\end{tabular} & $\begin{array}{c}\delta \\
\delta \\
\delta \\
\delta\end{array}$ & & - & $\sim$ \\
\hline 8 & $\vec{\nabla}$ & 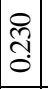 & 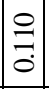 & $\begin{array}{l}\frac{?}{5} \\
0 \\
\end{array}$ & 8 & \begin{tabular}{|l|} 
\\
\\
\\
\end{tabular} & $\begin{array}{l}0 \\
\\
0 \\
0\end{array}$ & \begin{tabular}{|c|} 
\\
กิ \\
0
\end{tabular} & 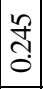 & \begin{tabular}{l}
$\mathfrak{2}$ \\
\hdashline \\
0
\end{tabular} & ठิ & क़? & 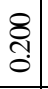 & | & -1 & 0 & -1 \\
\hline 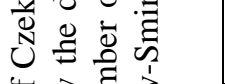 & $\frac{z}{5}$ & đิ & 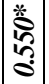 & 号 & $\frac{9}{9}$ & तิ & $\frac{8}{0}$ & $\mid \begin{array}{c}8 \\
\tilde{\pi} \\
0\end{array}$ & $\frac{2}{0}$ & 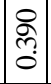 & 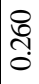 & $\frac{\infty}{0}$ & & $\infty$ & $N$ & $\sim$ & 0 \\
\hline 2 & \begin{tabular}{|l|} 
\\
$z$ \\
$z$
\end{tabular} & 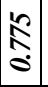 & กิ & 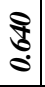 & @্ণি & 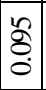 & $\begin{array}{l}\stackrel{0}{0} \\
\vdots \\
0\end{array}$ & $\left|\begin{array}{l}8 \\
\vdots \\
0\end{array}\right|$ & $\stackrel{?}{\stackrel{8}{8}}$ & 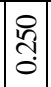 & 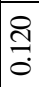 & 1 & $\simeq$ & $=$ & $N \mid$ & - & 0 \\
\hline . & $\sum_{\Sigma}^{\Xi}$ & $\mid \begin{array}{l}0 \\
\vdots \\
0 \\
0\end{array}$ & $\mid \begin{array}{l}0 \\
\& \\
0 \\
0\end{array}$ & 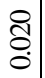 & 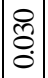 & 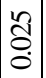 & 우 & $\mid$\begin{tabular}{l}
$:$ \\
\hdashline \\
\hdashline
\end{tabular} & $\frac{8}{0}$ & 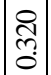 & I & $m$ & in & $m$ & $N \mid$ & $N$ & $\nabla$ \\
\hline . & $\sum_{\Sigma}^{800}$ & $\mid \begin{array}{c}0 \\
0 \\
0 \\
0\end{array}$ & 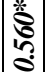 & $\begin{array}{l}0 \\
0 \\
0 \\
0\end{array}$ & $\mid$\begin{tabular}{l}
0 \\
\hdashline \\
0 \\
0
\end{tabular} & $\begin{array}{l}8 \\
8 \\
0\end{array}$ & 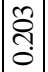 & ڤેণ & 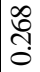 & 1 & 0 & 10 & \pm & a & $N \mid$ & $N$ & r \\
\hline 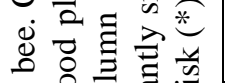 & $\overline{\mathbf{\pi}}$ & 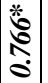 & సิ & 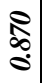 & $\mid \begin{array}{l}8 \\
8 \\
0 \\
0\end{array}$ & $\underset{0}{\stackrel{0}{0}}$ & $\frac{\bar{\sigma}}{0}$ & $\mid \begin{array}{l}8 \\
2 \\
2 \\
8\end{array}$ & 1 & $\bar{\lambda}$ & 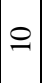 & $\underset{\sim}{\infty}$ & 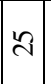 & $\infty$ & $N \mid$ & $N$ & $r$ \\
\hline . & 导 & 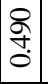 & 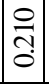 & ลี & $\begin{array}{c}\otimes \\
0 \\
0\end{array}$ & $\begin{array}{l}0 \\
8 \\
0 \\
0\end{array}$ & $\stackrel{0}{=}$ & I & ల & $\infty$ & $n$ & I & 2 & 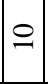 & $N$ & - & $\infty$ \\
\hline . & $\tilde{\tilde{E}}$ & $\begin{array}{c}8 \\
\delta \\
0\end{array}$ & $\frac{1}{0}$ & $\begin{array}{l}8 \\
0 \\
0\end{array}$ & $\begin{array}{l}8 \\
8 \\
0 \\
0\end{array}$ & $\because$ & 1 & $=$ & 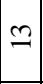 & $\infty$ & $m$ & $\infty$ & -1 & $N$ & -1 & 0 & $\nabla$ \\
\hline 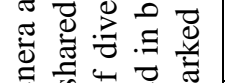 & שَّ & $\begin{array}{c}0 \\
\delta \\
0\end{array}$ & $\frac{8}{0}$ & $\begin{array}{l}5 \\
0 \\
0\end{array}$ & $\begin{array}{l}\stackrel{2}{0} \\
0 \\
0\end{array}$ & 1 & -1 & $\nabla$ & 6 & $m$ & $N$ & $m$ & $\nabla$ & $m$ & -1 & 0 & - \\
\hline 4 & ڤ్ & $\begin{array}{l}0 \\
0 \\
0\end{array}$ & 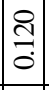 & 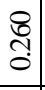 & 1 & $\nabla$ & $r$ & ה & 寺 & 9 & $r$ & 잉 & \pm & in & $N$ & - & $r$ \\
\hline - & $\Xi$ & ฐి & 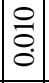 & 1 & $\approx$ & in & 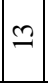 & $\hat{m}$ & in & ৯े & $\varrho$ & $\bar{\lambda}$ & $\tilde{\lambda}$ & $=$ & $N$ & $N$ & $\infty$ \\
\hline 87 & $\overrightarrow{\mathrm{z}}$ & : & 1 & \pm & $a$ & $m$ & in & 의 & \pm & $\simeq$ & $n$ & $r$ & 이 & $m$ & $N$ & $N$ & $m$ \\
\hline 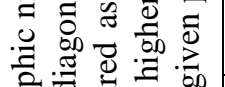 & $\overrightarrow{\mathbf{E}}$ & । & \pm & $\bar{n}$ & オ & in & \pm & 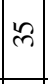 & $n$ & \% & $a$ & $\approx$ & $\approx$ & $=$ & $N$ & $N$ & $\infty$ \\
\hline 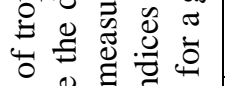 & $\overline{n^{2}}$ & 6 & $\simeq$ & 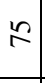 & ஜి & 0 & $\simeq$ & 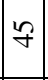 & $\approx$ & ले & $\cong$ & $\bar{m}$ & ते & 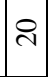 & $\sim \mid$ & $N$ & $\infty$ \\
\hline 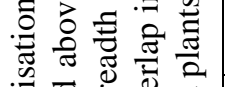 & $\mathbf{I}$ & $\stackrel{\vec{m}}{\mathrm{~d}}$ & $\sqrt{n}$ & I & $\underset{\sim}{\sim}$ & 3. & 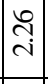 & 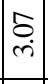 & is & $\begin{array}{l}\infty \\
\stackrel{i}{r}\end{array}$ & $\stackrel{2}{\circ}$ & $\frac{O}{m}$ & $\frac{m}{m}$ & $\mid \begin{array}{l}\vec{\infty} \\
i\end{array}$ & | & $\stackrel{\infty}{0}$ & - \\
\hline 4 & 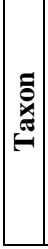 & 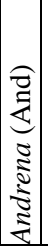 & 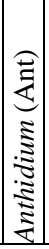 & 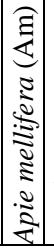 & $\left|\begin{array}{c}\Xi \\
0 \\
0 \\
\vdots \\
\vdots \\
\vdots \\
\vdots \\
\vdots \\
0\end{array}\right|$ & 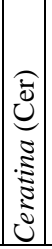 & 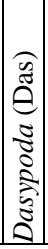 & 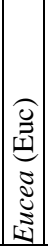 & 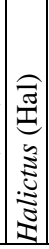 & 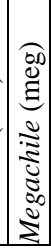 & 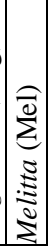 & $\left|\begin{array}{c}\mathfrak{z} \\
0 \\
z \\
z \\
\tilde{z} \\
\mathfrak{z} \\
\vdots \\
0 \\
z\end{array}\right|$ & 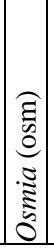 & 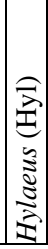 & 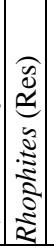 & 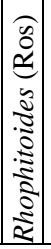 & $\underset{3}{3}$ \\
\hline
\end{tabular}


fed mainly on Cychorium intybus, Salvia nemorosa and Echium vulgare, which were not frequently recorded for the other taxa. This genus was almost the only visitor to Sambucus ebulus, Crepis paludosa and Myosotis intermedia, which played a relatively important role in its diet.

High niche overlap indices were additionally obtained for 12 pairs of bee taxa, but the distributions of use of individual plant species were not significantly similar. This means that these wild bee genera visited similar plant species but did so with different intensity.

A high degree of niche overlap was demonstrated for the genera Andrena, Halictus s.l., Nomada and the honey bee. The highest number of records for these taxa was for oilseed rape and radish. Apis mellifera and Halictus s.l. were, however, characterised by a higher proportion of visitations of summer-blooming sunflower crops, with the honey bee additionally feeding on flowers of Phacelia. The genus Nomada followed a very diversified $\operatorname{diet}\left(H^{\prime}=3.10\right)$, with a higher proportion of visitations of plants of less importance for the other genera.

The composition of food plants for the genus Anthidium was most similar to those of the genera Megachile and Osmia. The greatest proportion of its diet consisted of Lamium purpureum, Centaurea orientalis and Alkanna strybnyi. Megachile was specifically characterised by feeding on the genus Lamium, but the staples of its diet were Centaurea salonitana and 14 species of plants not visited by Osmia and Anthidium bees. The genus Osmia was most often reported from Lamium album, Erodium cicutarium and Papaver rhoeas, which accounted for small differences between the niches of Anthidium and Megachile.

The genus Eucera had high niche overlap indices with the genera Halictus s.l., Melitta, Rophites, Rophitoides and the honey bee. The latter three genera had very similar trophic bases. The genus Halictus s.l., compared to Eucera, was keener to use crops of Helianthus annuus, Raphanus sativus and Carduus acanthoides. Oilseed rape constituted a similarly high proportion of the diets of both genera, while the diet of Eucera bees was characterised by higher proportions of 12 plant species visited by both taxa.

Rophites and Rophitoides were recorded almost exclusively from the alfalfa field, accounting for more than $95 \%$ of the records for both these taxa and resulting in a high index of overlap of their very narrow niches $\left(H^{\prime}=0.06\right.$ and $\left.H^{\prime}=0.08\right)$. Medicago sativa was often chosen by the genus Melitta, but its slightly wider niche $\left(\mathrm{H}^{\prime}=0.76\right)$ made its pattern of resource exploitation different from the two genera mentioned above. 


\section{Competition in assemblages}

The co-occurrence of taxa in the study sites was analysed (Fig. 1). The highest indices of temporal niche overlap (Czekanowski's Index) were obtained for crops of sunflower, oilseed rape and radish. This means that a number of bee genera were seen foraging on abundantly growing crops at the same time. The temporal niche overlap index for the alfalfa field is more than $50 \%$ lower and comparable to those obtained for the cluster of semi-natural habitats, indicating a rather high degree of separation during flights over this plantation.
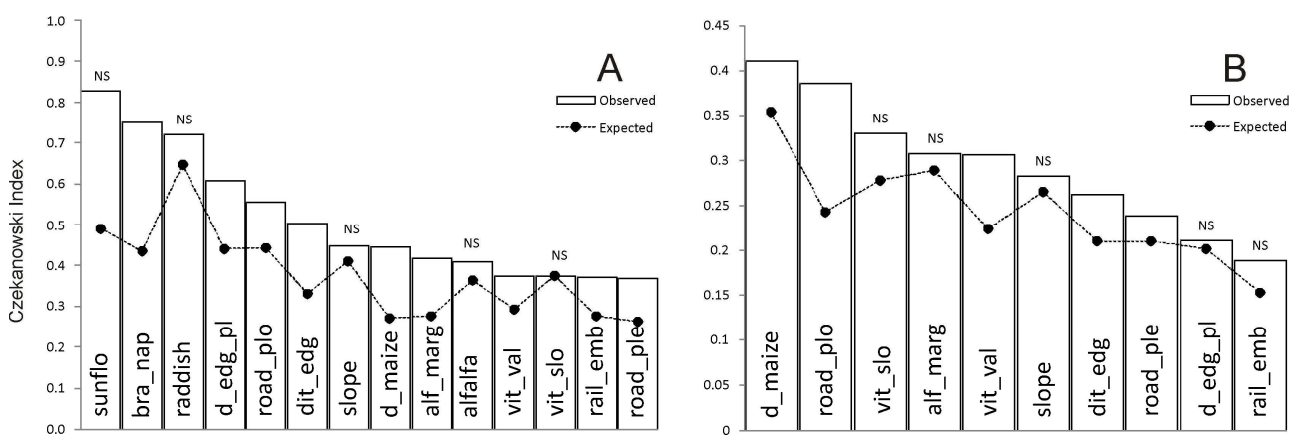

Fig. 1. Expected and observed temporal (A) and trophic (B) niche overlap, expressed as Czekanowski's Indices, at individual study sites in Bulgaria. See Table 1 for a key to the codes. "NS" indicates lack of statistical significance.

The niche overlap index calculated for food resources assumed lower values than those obtained for temporal niches at the study sites (Fig 1B). Thus, the bees co-occurred in time but used different trophic bases. The crop fields are not included in the graphs since randomisation was not possible in this case owing to the lack of variability in the food base. Czekanowski's Index for these sites was 1.

All actual values of trophic and temporal niche overlap were equal to or significantly higher than those expected in the null model. This would indicate that competition was not a determinant of the structure of pollinators visiting the plants at individual sites. If competition significantly influenced resource division, actual niche overlap ought to have been lower than the random value. 


\section{Intensity of plant visitations by bees}

The mean number of visitations of food plants during the season varied widely for the honey bee (Fig. 2), while the intensity of food plant visitations by wild bees grew systematically, stabilising in the second half of May. The visitation count in the first half of September was stable and low.

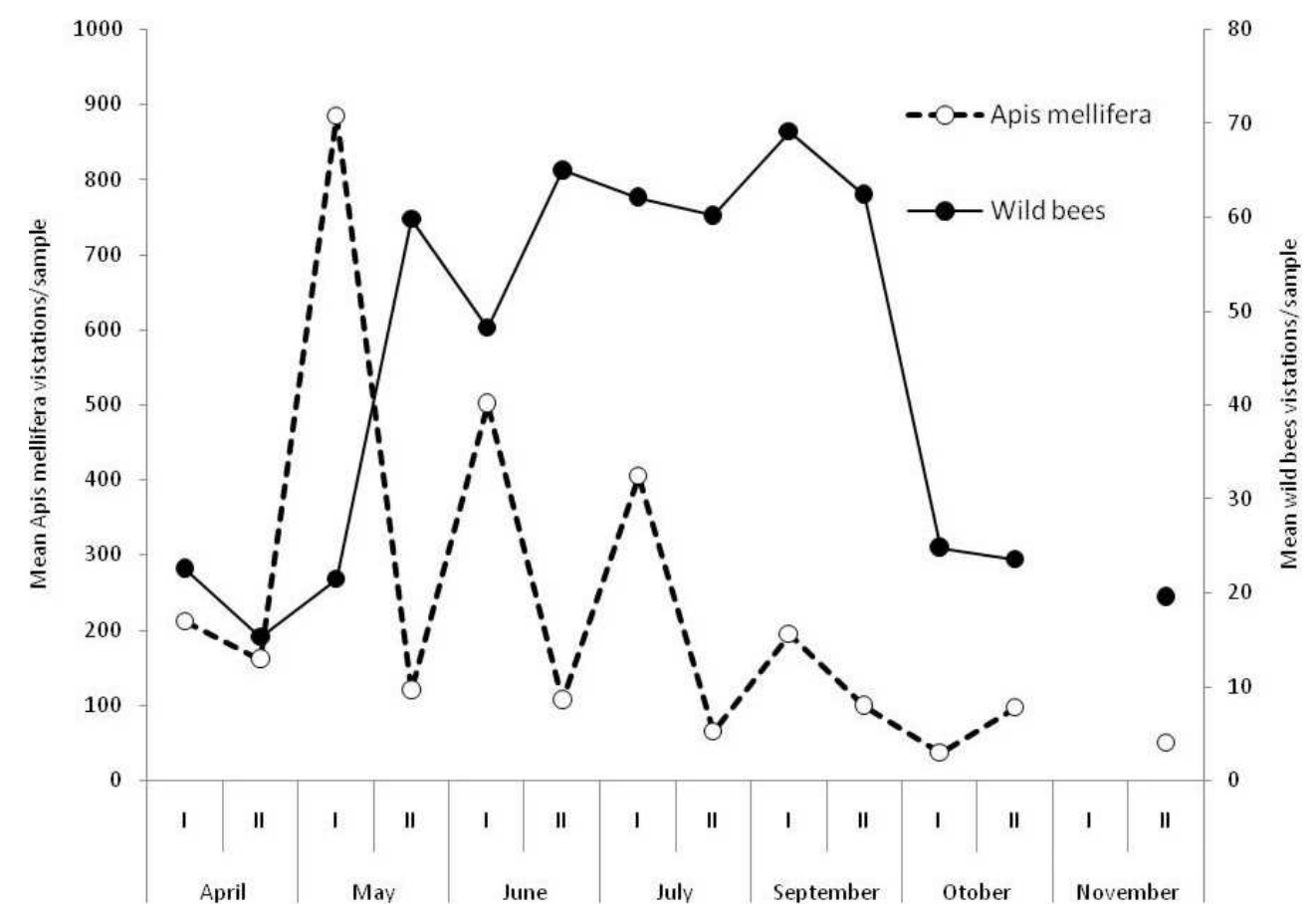

Fig. 2. Mean count of recorded visitations of flowers by the honey bee and wild bees per transect. The means were calculated for all years of the study (1987-1991).

The NMDS method was used to analyse the similarity of activity of individual bee genera (Tab. 4) in the two clusters of habitats of interest, i.e. crop fields and refuge habitats (Fig. 3). With regard to crops, there was a high similarity in pollinator activity for oilseed rape and radish, and a somewhat less distinct similarity for phacelia and sunflower. The alfalfa plantation was quite different from both crop fields and refuge habitats with regard to pollinator activity, with the greatest differences noted between this site and the phacelia plantation. This finding was probably associated with distinct conditions in this plantation, which had been developing for many years, and the fact that some pollinators nested there. 


\begin{tabular}{|c|c|c|c|c|c|c|c|c|c|c|c|c|c|c|c|c|c|}
\hline 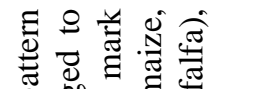 & $\stackrel{2}{2}$ & road_pl & {$\left[\begin{array}{lll}0 \\
0 \\
i\end{array}\right]$} & $\begin{array}{l}8 \\
0\end{array}$ & & 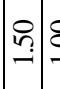 & & 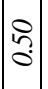 & & & 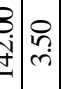 & $\stackrel{8}{=}: 0$ & $\ln$ & $i$ & $\begin{array}{l}8 \\
\vdots \\
0 \\
0\end{array}$ & & 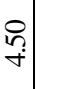 \\
\hline 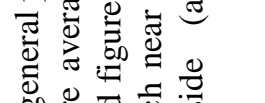 & \pm & slope & : & 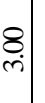 & 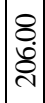 & & & & : & & & $\begin{array}{lll}8 & 8 \\
& 8 \\
\end{array}$ & $\begin{array}{l}8 \\
\dot{\oplus}\end{array}$ & $\begin{array}{l}8 \\
\dot{\sim i}\end{array}$ & & ल. & $\stackrel{8}{-}$ \\
\hline 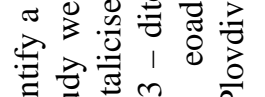 & $\approx$ & vit_slo & $\mid$ & : & & ר. & & & & & & $\begin{array}{c}8 \\
i\end{array} \mid$ & $\mid \begin{array}{l}0 \\
i \\
i\end{array}$ & 8 & & : & 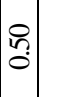 \\
\hline 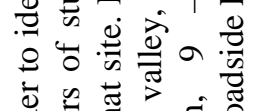 & $\mathcal{I}$ & sunflo & $\stackrel{0}{n}$ & & & 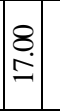 & & & & & $\begin{array}{l}8 \\
8 \\
0\end{array}$ & & & & & & 8 \\
\hline 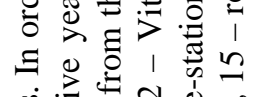 & & d_edg_pl & $\begin{array}{c}8 \\
0 \\
-0\end{array}$ & 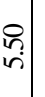 & & $\begin{array}{c}8 \\
i n\end{array}$ & & & † & & $\hat{n}$ & $\mid \begin{array}{l}8 \\
\dot{4} \\
\dot{4}\end{array}$ & $\begin{array}{lll}8 & 8 \\
\infty & 8 & \\
1\end{array}$ & $\mid \begin{array}{l}8 \\
\dot{+} \\
\dot{+}\end{array}$ & & & 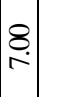 \\
\hline 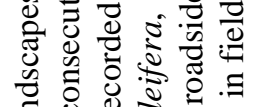 & $\mathcal{E}$ & radish & $\left|\begin{array}{ll}8 \\
8 \\
\infty \\
\infty\end{array}\right|$ & & 赵 & & & & & âd & 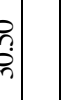 & & $\ln$ & 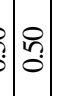 & & & 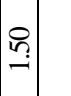 \\
\hline $\begin{array}{l}1=\overline{3} \\
0 \\
5 \\
5\end{array}$ & & alf_marg & $\left|\begin{array}{c}\vec{b} \\
\dot{f}\end{array}\right|$ & ֻి. & $\begin{array}{l}8 \\
\square \\
i n \\
n\end{array}$ & & & & is & & 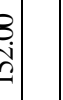 & $\widehat{\overbrace{}}$ & 8 & $\hat{\overbrace{}}$ & & 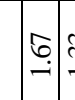 & ڤి \\
\hline 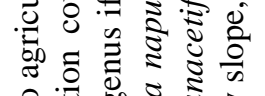 & 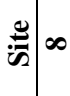 & road_ple & $\grave{3}$ & 它| & & 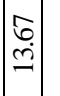 & $\stackrel{m}{\infty}$ & & $\overbrace{\infty}^{\infty}$ & ? & : & $\widehat{్}$ & $\stackrel{8}{\rightarrow}$ & id & & & 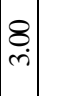 \\
\hline.$\overline{\frac{2}{5}}$ & r & phacelia & & & & 8 & & & & & & & & & & & \\
\hline$\stackrel{\partial}{\partial}$ & 6 & dit_edg & $\left|\begin{array}{c}8 \\
\infty\end{array}\right|$ & : & & 8 & ?ִ? & ?ุ: & 8 & & ל. & : & $\delta_{\infty}^{8}$ & 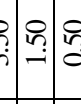 & ?. & & $\begin{array}{l}8 \\
\dot{m}\end{array}$ \\
\hline 3 & in & rail_emb & $\begin{array}{l}8 \\
-9\end{array}$ & 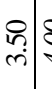 & 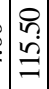 & 8 & & & 8 & & : & $\underset{i}{\stackrel{i}{i}}$ & $\mathbb{i}_{i}^{8}$ & b. & & & 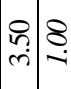 \\
\hline $\begin{array}{ll}0 & \vec{w} \\
b & 1\end{array}$ & 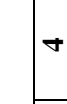 & alfaalfa & בิ & હે. & fi. & f. & ֻุ| & & $\approx$ & & $\begin{array}{l}q \\
q \\
\infty \\
\infty\end{array}$ & 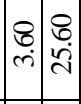 & 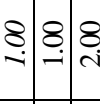 & & 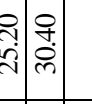 & ชิ่ & \\
\hline$\Xi$ & $m$ & d_maize & $\left|\begin{array}{c}8 \\
\dot{m} \\
m\end{array}\right|$ & $\stackrel{m}{0}$ & & $\stackrel{0}{\circ}$ & & के & $\widehat{c}$ & & : & 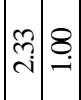 & 80 & $\dot{\hat{b}}$ & & 灾 & $\mid \begin{array}{l}8 \\
+ \\
\end{array}$ \\
\hline $\mathscr{\Xi}$ & N & val_vit & $\left|\begin{array}{c}8 \\
\text { in }\end{array}\right|$ & - & & $\stackrel{\overbrace{}}{\sim}$ & $\stackrel{\curvearrowright}{0}$ & & 8 & & 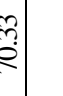 & மீ. & مُ & | & & 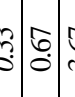 & 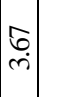 \\
\hline 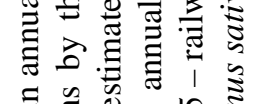 & - & bra_nap & 官 & & 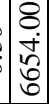 & : & & & 5 & & 焉 & & $\mid$ & : & & & $\left|\begin{array}{l}0 \\
i \\
i \\
c\end{array}\right|$ \\
\hline 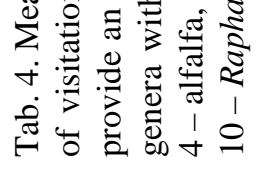 & 㺼 & & 离: & & & 0 & 0 & & 015 & & & 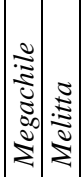 & 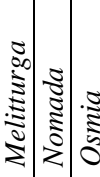 & 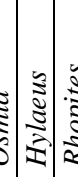 & 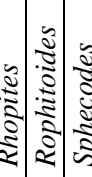 & 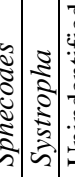 & 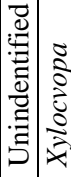 \\
\hline
\end{tabular}




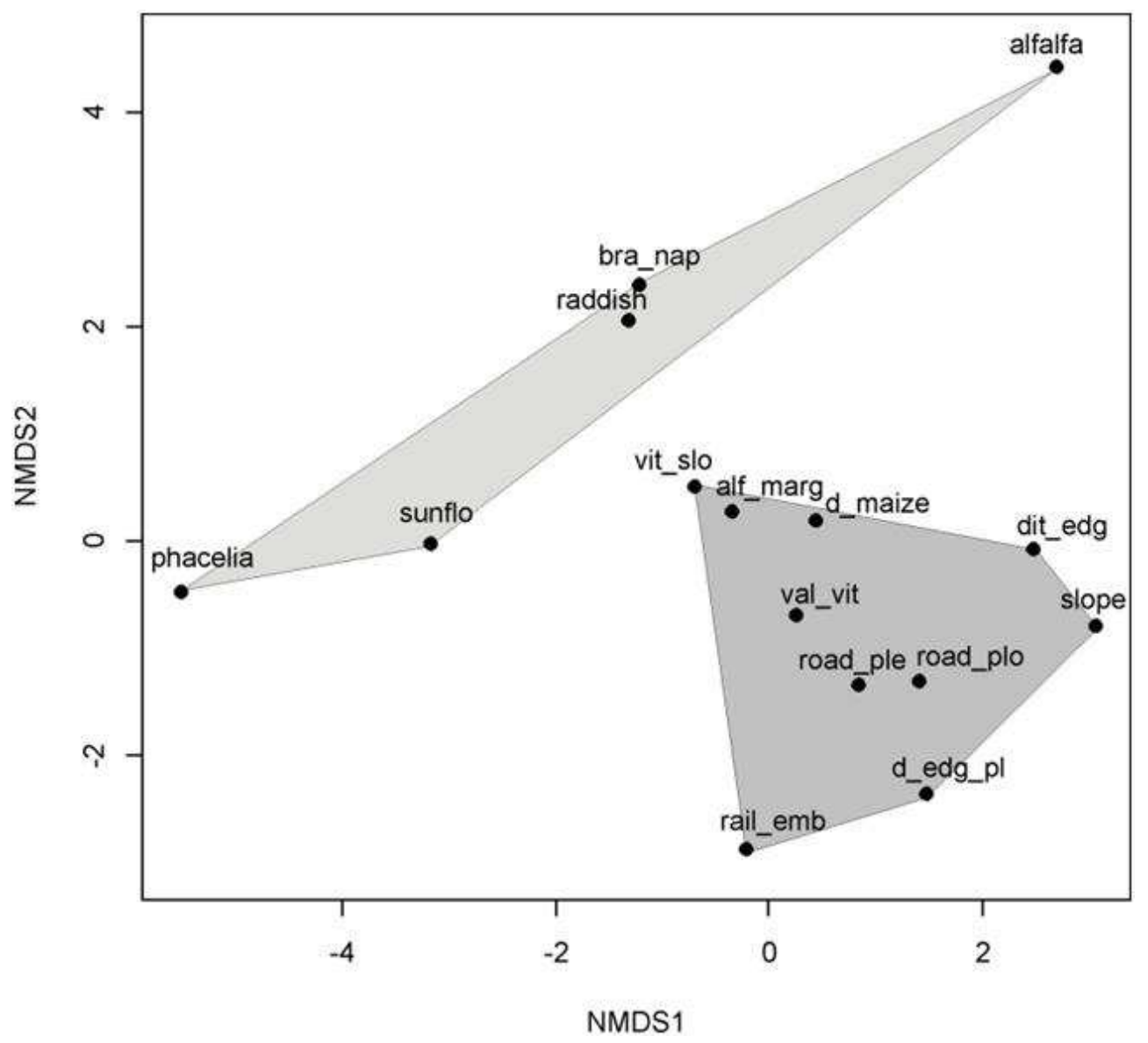

Fig. 3. NMDS ordination graph ( $\mathrm{k}=2$, Distance: Bray-Curtis) for sites with regard to visitation count by individual genera. The significance of division was verified with the MRPP test $(\mathrm{p}=0.008)$. The dashed line connects groups of crop fields and semi-natural sites. See Table 1 for site codes.

Among the refuge habitats, the sites Vit slope, Alfalfa margin and Ditch near maize demonstrated considerable similarity to the crops of oilseed rape and radish. All 10 seminatural sites were also more homogeneous as a cluster in terms of pollinator activity than the crop fields.

MRPP analysis found significant differences between the clusters with regard to the activity of individual bee genera. Indicator values were calculated in order to determine which genera were responsible for the significance of the differences (Tab. 5). For the crop fields, one distinctive species, Apis mellifera, was significant. For the refuge habitats, there 
were 6 significant characteristic genera: Systropha, Anthidium, Megachile, Hylaeus, Dasypoda, Halictus s.l.

Tab. 5. Characteristic genera (indicator values and significance) for two arbitrary clusters of crop fields and refuge habitats, calculated with the IndVal method.

\begin{tabular}{|c|c|c|c|}
\hline Genus & Cluster & Indicator value & Probability \\
\hline Apis & Crop field (c ) & 0.8735 & 0.021 \\
\hline Systropha & Refuge habitats (s) & 0.9959 & 0.002 \\
\hline Anthidium & Refuge habitats (s) & 0.9857 & 0.001 \\
\hline Megachile & Refuge habitats (s) & 0.9227 & 0.003 \\
\hline Hylaeus & Refuge habitats (s) & 0.9091 & 0.002 \\
\hline Dasypoda & Refuge habitats (s) & 0.8000 & 0.016 \\
\hline Halictus & Refuge habitats (s) & 0.7161 & 0.018 \\
\hline
\end{tabular}

The arrangement of sites according to the first dimension in the ordination graph can be attributed to increasing density of wild bees and a distinct fall in the density of the honey bee (Tab. 6). These sites mainly represent refuge habitats and also include the alfalfa field. The analysis also confirms the division of the sites into two clusters as their co-ordinates in the NMDS chart relatively well reflect their placement in the crop field or refuge habitat cluster. The trophic niche overlap index decreases from the crop field habitats to the refuge habitats. The site co-ordinates of the second dimension of the NMDS ordination were associated with increasing overlap of trophic niches.

Tab. 6. Spearman's coefficients of non-parametric rank correlation for MDS dimensions and for characteristics of the study habitats.

\begin{tabular}{|c|c|c|}
\hline Variables & Spearman's R & p \\
\hline NMDS1 \& Habitat type & 0.589188 & 0.020821 \\
\hline NMDS1 \& Trophic niche overlap & -0.57517 & 0.024883 \\
\hline NMDS1 \& Wild bee density & 0.564286 & 0.028433 \\
\hline NMDS1 \& Honey bee density & -0.75357 & 0.001178 \\
\hline NMDS2 \& Trophic niche overlap & 0.677103 & 0.005557 \\
\hline
\end{tabular}




\section{DISCUSSION, KEY FINDINGS AND CONCLUSIONS}

Showing a high visitation intensity on flowers for the honey bee, our results confirm the high potential competitiveness of this species relative to that of wild bees (STEFFANDEWENTER \& TSCHARNTKE 2000). Trophic niche overlap between genera was mostly (75\% of pairs) a phenomenon of minor importance, reaching values below $25 \%$ of the overlap between resource use distribution. Bees' trophic niches at the generic level did not demonstrate significant overlap. Similar studies at species level (AGUIAR \& SANTOS 2007, AGUIAR et al. 2012) produced different results, with significantly lower niche overlap figures obtained for individual bee species than in random records. Thus, competition does not appear to play a significant role in pollinator assemblages at the generic level. If different bee genera feed on similar plant species, these species usually account for different proportions of their diets. Mean trophic niche overlap indices for the study sites decreased from the crop field habitats to the refuge habitats. This would suggest greater functional redundancy in the crop fields and functional complementarity in the refuge habitats (BLÜTHGEN \& KLEIN 2011). Bee activity in the crop fields differed significantly from their pattern of activity in the refuge habitats. Crops were not a significant food source for many species of the genera Anthidium, Hylaeus, Megachile, Osmia, Dasypoda, Systropha and Halictus s.l. These genera were typically associated with refuge habitats. The decreasing proportion of semi-natural habitats in the agricultural landscape may pose a threat to these taxa and result in changes in their contribution to the structural indices of diversity and species richness. The question arises as to whether these genera are able to switch to food offered by abundantly blooming crops with the decreasing refuge habitat cover of the agricultural landscape. The attractiveness of flowering crops (agricultural matrix) depends, among many other factors, on bees' foraging ranges and the density of flower resources currently available in their surroundings (RANDS \& WHITNEY 2010, 2011). The simple density-dependent process of bees switching to utilise mass flowering crops could be modified by the high habitat fidelity of these insects and neophobia, i.e. resource fidelity (FrANZEN et al. 2009, WiLliams \& KREMEN 2007). BOMMARCO et al. (2010) showed that different responses of wild bees to habitat loss and increasing crop fields area depend on species body size and diet breadth. According to their results, smallsized generalist bee species are more prone to habitat loss than large-sized bee species. Moreover, specialised bee species were more affected than generalists. Body size and dietary constraints, which force bees to remain in their primary habitat, could explain why some species stay in the refuge habitats even if flowering crops are present nearby. Foraging ranges of wild bees correlate positively with body size (GATHMAN \& TSCHARNTKE 2002), so the utilisation of abundant but distant resources may be limited for some small species, especially if they have narrow trophic niches. Furthermore, generalist bees respond strongly to mass flowering crops, which is why plants in semi-natural habitats 
may experience transient pollination dilution (HOLZSCHUH et al. 2011). Of course, some bee species could be absent from fields simply because of lack of phenological overlap with crop flowering. The high degree of overlap of temporal niches in the crops is due to the short period and large-scale pattern of blooming. Plantations offer a very rich trophic base and there is no observed competition for the resources of massively blooming annual crops. There were some distinct differences regarding the utilisation of flower resources on mass flowering crops. Phacelia flowers were visited almost exclusively by Apis mellifera, while the alfalfa field supported a high diversity of wild bee genera. The unique character of the alfalfa field was also noted by RoLLIN et al. (2013). In their work they showed that flowering alfalfa fields are attractive floral resources for all bee groups: Apis mellifera, bumblebees and wild bees.

Species within one genus are closer to one another in terms of ecology and morphology, which may result in greater competition within a genus than between genera. The trophic niche overlap index, expressed as Czekanowski's Index, decreases from the crop field habitats to the refuge habitats, which is associated with increasing diversity of available food. The density of wild bees increases and the density of the honey bee decreases. It is not known whether Apis mellifera is usually the most abundant bee species on flowering crops. Nonetheless, wild bees are generally important pollinators of mass flowering crops, not only substituting for honey bees, but actually increasing crop yields (GARIBALDI et al. 2011, 2013). Therefore it is important to broaden our knowledge about plant resources used by wild bees in marginal habitats in agricultural landscapes to sustain the local and regional diversity of this group of pollinators as well as a high level of pollination services of crops.

\section{REFERENCES}

Aguiar C.M.L., Santos G.M. de M. 2007. Floral Resource Partitioning by Social Wasps (Hymenoptera: Vespidae) and Bees (Hymenoptera: Apoidea) in an Area of Caatinga in Brazil. Neotropical Entomology 36(6): 836-842. (in Portuguese)

Aguiar C.M.L., Santos G.M. de M., Martins C.F., Presley S.J. 2013. Trophic niche breadth and niche overlap in a guild of flower-visiting bees in a Brazilian dry forest. Apidologie 44(2): $153-162$.

AtANASSOv N. 1960. Untersuchungen über die Arten aus der Familie Halictidae (Hymenoptera) in Bulgariens und der Balkanhalbinsel. Izvestiya na Zoologicheskiya institut s muzei 9: 315-338.

AtANASSOv N. 1962a. Untersuchungen über die Arten von der Gattung Xylocopa LATR. (Hymenoptera, Apoidea) in Bulgarien und auf der Balkanhalbinsel. Izvestiia na Zoologicheskiya Institut s Muzei 11: 167-180.

ATANASSOv N. 1962b. Untersuchungen über die systematik und die Ökologie der Hymenopterenarten aus dem Gebiet von Petrič (Südwestbulgarien). Izvestiia na Zoologicheskiya Institut s Muzei 12: $109-172$. 
AtAnAssov N. 1965. Hymenoptera von der Insel Thasos. Izvestiia na Zoologicheskiya Institut s Muzei 19: 85-99.

AtAnAssov N. 1972a. Hymenopteraarten im Westlichen "Stara Planina“ - Gebirge. I. Izvestiia na Zoologicheskiya Institut s Muzei 35: 179-228.

ATANASSOV N. 1972b. Hymenopteraarten im Westlichen „Stara Planina“ - Gebirge. II. Izvestiia na Zoologicheskiya Institut s Muzei 36: 23-59.

AtANASSOv N. 1974. Hummeln und Schmarotzerhummeln (Bombus LATR., Psithyrus LEP., Hym.) von dem Mittleren und dem Östlichen Balkangebirge. Izvestiia na Zoologicheskiya Institut s Muzei 41: 107-121.

Atanassov N., Vassileva E. 1981. Bees - pollinators (Hymenoptera, Apoidea) of alfalfa in the region of Pleven. III. Seasonal dynamics of the species. Plant Science 18(3): 102-109.

BANASZAK J. 1980. Studies on methods of censusing the numbers of bees (Hymenoptera, Apoidea). Polish Ecological Studies 6(2): 355-366.

BANASZAK J. 1983. Ecology of bees (Apoidea) of agricultural landscape. Polish Ecological Studies 9(4): 421-505.

Banaszak J., Dochkova B. 2014. Bees (Hymenoptera, Apoidea, Apiformes) in the agricultural lanscape of Bulgaria: species diversity. Journal of Apicultural Science 58(1): 29-49.

BlÜthgen N., Klein A.-M. 2011. Functional complementarity and specialisation: The role of biodiversity in plant-pollinator interactions. Basic and Applied Ecology 12(4): 282-291.

Bommarco R., Biesmeijer J.C., Meyer B., Potts S.G., Pöyry J., Roberts S.P.M., SteffanDEWENTER I., ÖCKINGER E. 2010. Dispersal capacity and diet breadth modify the response of wild bees to habitat loss. Proceeding of the Royal Society of London B 277: 2075-2082.

Boulinier T., Nichols J.D., SAuer J.R., Hines J.E., Pollock K.H. 1998. Estimating species richness: the importance of heterogeneity in species detectability. Ecology 79(3): 1018-1028.

Castro-Arellano I., Lacher T.E. Jr., Willig M.R., Rangel T.F. 2010. Assessment of assemblagewide temporal-niche segregation using null models. Methods in Ecology and Evolution 1(3): 311-318.

Dimitrov P. 1990. Effect of the density of the wild bees (Hymenoptera, Apoidea) on the percentage of pollination of lucerne blossoms. Plant Science 27(6): 23-27.

Dimitrov P., Dimitrova Z. 1991a. Studies of the provision of alfalfa fields for seed production with pollinating bees (Hymenoptera Apoidea) in conditions of north-eastern Bulgaria. Agricultural Science 29(4-6): 90-93.

Dimitrov P., Dimitrova Z. 1991b. A rapid method of determining the density of wild bees (HyMENOPTERA, Apoidea) in lucerne grown for seed. Plant Science 28(3-6): 118-119.

Dochkova B. 1981a. Alfalfa Megachile rotundata F. (= pacifica Pz.) (Hymenoptera, Megachilidae). I. Morphological and biological characteristics. Plant Science 18(8): 91-98.

Dochкova B. 1981b. Bees-pollinators (Hymenoptera, Apoidea) of alfalfa in the region of Pleven. V. Economic importance. Plant Science 18(5): 148-155.

DochKova B. 1982a. Alfalfa leaf-cutting bee Megachile rotundata F. (= pacifica Pz.) (Hymenoptera, Megachilidae). II. Building and pollination activity. Plant Science 19(1): 112-118. 
Dochkova B. 1982b. The alfalfa leaf cutter-bee (Megachile rotundata F.) (= pacifica Pz.) (Hymenoptera, Megachilidae). III. Natural pests and possibilities for their control. Plant Science 19(2): 118-127.

DufrêNE M., LeGENDRE P. 1997. Species assemblages and indicator species: the need for a flexible asymmetrical approach. Ecological Monographs 67(3): 345-366.

Feinsinger P., Spears E.E., Poole R.W. 1981. A simple measure of niche breadth. Ecology 62(1): $27-32$.

FrANZÉn M., LARSSON M., NiLSSON S.G. 2009. Small local population sizes and high habitat patch fidelity in a specialised solitary bee. Journal of Insect Conservation 13(1): 89-95.

Garibaldi L.A., Steffan-Dewenter I., Kremen C., Morales J.M., Bommarco R., Cunningham S.A., Carvalheiro L.G., Chacoff N.P., DudenhöFfer J.H., Greenleaf S.S., Holzschuh A., Isaacs R., Krewenka K., Mandelik Y., MaYfield M.M., Morandin L.A., PotTs S.G., Ricketts T.H., Szentgyörgyi H., Viana B.F., WestPhal C., Winfree R., Klein A.M. 2011. Stability of pollination services decreases with isolation from natural areas despite honey bee visits. Ecology Letters 14(10): 1062-1072.

Garibaldi L.A., StefFan-Dewenter I., Winfree R., Aizen M.A., Bommarco R., Cunningham S.A., Kremen C., Carvalheiro L.G., Harder L.D., Afik O., Bartomeus I., Benjamin F., Boreux V., Cariveau D., Chacoff N.P., Dudenhöffer J.H., Freitas B.M., Ghazoul J., Greenleaf S., Hipólito J., Holzschuh A., Howlett B., Isaacs R., Javorek S.K., Kennedy C.M., Krewenka K.M., Krishnan S., Mandelik Y., Mayfield M.M., MotZKe I., Munyuli T., Nault B.A., Otieno M., Petersen J., Pisanty G., Potts S.G., Rader R., Ricketts T.H., RundlöF M., Seymour C.L., Schüepp C., Szentgyörgyi H., TAKi H., Tscharntke T., Vergara C.H., Viana B.F., Wanger T.C., Westrhal C., Williams N., Klein A.M. 2013. Wild Pollinators Enhance Fruit Set of Crops Regardless of Honey Bee Abundance. Science 339(6127): 1608-1611.

Gathmann A., TscharnTKE T. 2002. Foraging ranges of solitary bees. Journal of Animal Ecology 71(5): 757-764.

Gotelli N.J., EnTSMinger G.L. 2001. EcoSim: Null models software for ecology. Version 7.0. Acquired Intelligence Inc. \& Kesey-Bear. Internet: http://homepages.together.net/ gentsmin/ ecosim.htm

Gotelli N.J., Graves G.R. 1996. Null Models in Ecology. Smithsonian Institution Press, Washington, DC.

Holzschun A., Dormann C.F., TscharntKe T., StefFan-Dewenter I. 2011. Expansion of massflowering crops leads to transient pollinator dilution and reduced wild plant pollination. Proceeding of the Royal Society of London B 278: 3444-3451.

Holzschun A., DudenhöFfer J.-H., TscharntKe T. 2012. Landscapes with wild bee habitats enhance pollination, fruit set and yield of sweet cherry. Biological Conservation 153(): 101-107.

KenNedy C.M., Lonsdorf E., NeEl M.C., Williams N.M., RicketTS T.H., Winfree R., Bommarco R., Brittain C., Burley A.L., Cariveau D., Carvalheiro L.G., Chacoff N.P., Cunningham S.A., Danforth B.N., Dudenhöffer J.-H., Elle E., Gaines H.R., Garibaldi L.A., Gratton C., Holzschun A., Isaacs R., Javorek S.K., Jha S., Klein A.M., Krewenka K., Mandelik Y., Mayfield M.M., Morandin L., Neame L.A., Otieno M., Park M., Potts S.G., RundlöF M., Saez A., Steffan-Dewenter I., Taki H., Viana B.F., WestPhal C., Wilson J.K., Greenleaf S.S., KREMEN C. 2013. A global quantitative synthesis of local and landscape effects on wild bee pollinators in agroecosystems. Ecological Letters 16(5): 584-599. 
Klein A.-M., Brittain C., Hendrix S.D., Thorp R., Williams N., Kremen C. 2012. Wild pollination services to California almond rely on semi-natural habitat. Journal of Applied Ecology 49(3): 723-732.

LAWLOR L.R. 1980. Structure and stability in natural and randomly constructed competitive communities. American Naturalist 116(3): 394-408.

Le Féon V., Burel F., Chifflet R., Henry M., Ricroch A., Vaissière B.E., Baudry J. 2013. Solitary bee abundance and species richness in dynamic agricultural landscapes. [in:] F. BuREL, C. Lavigne, A.-C. Moonen, A. Ouin, S.L. Poggio (eds.). Landscape ecology and biodiversity in agricultural landscapes. Agriculture, Ecosystems \& Environment 166: 94-101.

Lentini P.E., Martin T.G., Gibbons P., Fischer J., Cunningham S.A. 2012. Supporting wild pollinators in a temperate agricultural landscape: Maintaining mosaics of natural features and production. Biological Conservation 149: 84-92.

MACQueEN J.B. 1976. Some methods for classification and analysis of multivariate observations. [in:] L.M. Le CAM, J. NeYMAN (eds.). Proceedings of $5^{\text {th }}$ Berkeley Symposium of Mathematical Statistics and Probability, Vol. 1. University of California Press, Berkeley, 281-297.

Mandelik Y., Winfree R., Neeson T., Kremen C. 2012. Complementary habitat use by wild bees in agro-natural landscapes. Ecological Applications 22(5): 1535-1546.

MARSHALl E.J.P. 2004. Agricultural landscapes: field margin habitats and their interaction with crop production. Journal of Crop Improvement 12(1-2): 365-404.

Morandin L.A., KREMEn C. 2013. Hedgerow restoration promotes pollinator populations and exports native bees to adjacent fields. Ecological Applications 23(4): 829-839.

NPGR [National Plant Genetic Resources] 1995. Bulgaria: Country Report to the International Technical Conference on Plant Genetic Resources (Leipzig 1996). National Plant Genetic Resources, Sadovo.

RANDS S.A., WhitNEY H.M. 2010. Effects of pollinator density-dependent preferences on field margin visitations in the midst of agricultural monocultures: A modelling approach. Ecological Modelling 221(9): 1310-1316.

RANDS S.A., Whitney H.M. 2011. Field margins, foraging distances and their impacts on nesting pollinator success. Plos One 6(10): e25971.

Rezende E.L., Lavabre J.E., Guimarães P.R., Jordano P., Bascompte J. 2007. Non-random coextinctions in phylogenetically structured mutualistic networks. Nature 448(7156): 925-928.

Rollin O., Bretagnolle V., Decourtye A., Aptel J., Michel N., Vaissière B.E., Henry M. 2013. Differences of floral resource use between honey bees and wild bees in an intensive farming system. Agriculture, Ecosystems \& Environment 179(1): 78-86.

SHANnON C.E. 1948. The Mathematical Theory of Communications. [in:] C.E. SHAnnon, W. WeAver (eds.). The mathematical theory of communication. University of Illinois Press, Urbana, 3-91.

SIEGEL S. 1956. Nonparametric Statistics for the Behavioral Sciences. McGraw-Hill Book Company Inc., New York.

TAshev A., Pancheva E. 2009. Systematic structure of the honey bearing plants of the Bulgarian flora. Forestry Ideas 15(1): 114-123 
Tashev A., Pancheva E. 2011. Conservation importance of the melliferous plants of the Bulgarian flora. Chornomorskiy Botanical Journal 7(2): 103-112.

Tuell J.K., Fiedler A.K., LANDis D., IsAaCs R. 2008. Visitation by wild and managed bees (Hymenoptera: Apoidea) to eastern U.S. native plants for use in conservation programs. Environmental Entomology 37(3): 707-718.

WiLliams N.M., KREMEN C. 2007. Resource distributions among habitats determine solitary bee offspring production in a mosaic landscape. Ecological Applications 17(3): 910-921.

Zimmerman G.M., Goetz H., MielKe JR. P.W. 1985. Use of an improved statistical method for group comparisons to study effects of prairie fire. Ecology 66(2): 606-611.

Received: 16 May 2015

Accepted: 12 June 2015 